\title{
Detection and mapping of soil liquefaction in the 2011 Tohoku earthquake using SAR interferometry
}

\author{
Kazuya Ishitsuka ${ }^{1}$, Takeshi Tsuji ${ }^{2}$, and Toshifumi Matsuoka ${ }^{1}$ \\ ${ }^{1}$ Department of Urban Management, Kyoto University, Kyoto, Japan \\ ${ }^{2}$ International Institute for Carbon-Neutral Energy Research (WPI-I2CNER), Kyushu University, Fukuoka, Japan
}

(Received December 27, 2011; Revised November 2, 2012; Accepted November 20, 2012; Online published January 28, 2013)

\begin{abstract}
We have identified areas of soil liquefaction by the analysis of surface changes caused by the 2011 Tohoku earthquake, using synthetic aperture radar (SAR) interferometry in the Kanto region of Japan. Changes in surface scattering properties were evaluated using phase-corrected coherence, computed from the reflective intensity (amplitude) of SAR data. Often, the loss of coherence (decorrelation) is simply considered to represent areas damaged from the disaster. However, temporal decorrelation could also be induced by ordinal surface cover change in addition to disaster damage. Therefore, we use a coherence change threshold to discriminate significant decorrelation caused by soil liquefaction from that produced by ordinal surface cover changes. Moreover, local surface displacements are estimated using phase information from the SAR data. Our results compare favorably with those from surveys of sand boils and aerial photography, showing that surface changes derived from SAR data are associated with soil liquefaction. Our results demonstrate that soil liquefaction occurred mainly near the waterfront along Tokyo Bay and the Tone River, and ground subsidence was widely distributed.
\end{abstract}

Key words: Soil liquefaction, SAR interferometry, interferometric coherence, temporal decorrelation, change detection, the 2011 Tohoku earthquake.

\section{Introduction}

The 2011 Tohoku earthquake $\left(M_{\mathrm{w}} 9.0\right)$ occurred northeast of the Japan Trench on 11 March with a rupture area as large as $500 \times 200 \mathrm{~km}$ (Ozawa et al., 2011), and was the fourth largest earthquake in the instrumental record. It was followed by numerous large aftershocks along that trench. In the Kanto region including Tokyo, about 350 $\mathrm{km}$ from the mainshock, seismic intensities of 5-lower to 6-lower were recorded (Fig. 1(a)) (Hoshiba et al., 2011). There were large areas of soil liquefaction, especially along Tokyo Bay and the Tone River, causing extensive damage to residential buildings and infrastructure (Bhattacharya et al., 2011; Yasuda and Harada, 2011; Senna et al., 2012). According to the geomorphologic classification of the Kanto region (Wakamatsu et al., 2005), areas around Tokyo Bay are mainly covered by filled land. Back marsh and natural levees are widely distributed along the upper Tone River, and delta and coastal lowland extend from the middle to the lower part of the river (Fig. 1(c)).

Soil liquefaction is usually investigated by field reconnaissance and aerial photography. Here, we successfully apply synthetic aperture radar (SAR) interferometry (InSAR), derived from satellite data, to identify and map soil liquefaction. Satellite-based remote sensing methods hold promise for providing broad and dense information on the Earth's surface (Massonnet and Feigl, 1998; Bürgmann et

Copyright (C) The Society of Geomagnetism and Earth, Planetary and Space Sciences (SGEPSS); The Seismological Society of Japan; The Volcanological Society of Japan; The Geodetic Society of Japan; The Japanese Society for Planetary Sciences; TERRAPUB.

doi:10.5047/eps.2012.11.002 al., 2000; Simons and Rosen, 2007) and have shown a potential advantage for investigating soil liquefaction (Atzori et al., 2012). To our knowledge, this study is the first application of InSAR analysis to mapping soil liquefaction caused by the 2011 Tohoku earthquake.

Soil liquefaction can cause decorrelation because the ground surface becomes wetter and structures may be tilted. We derive such changes of ground surface properties and thereby identify soil liquefaction areas by quantifying the degree of decorrelation, using phase-corrected coherence information based on SAR amplitude data. Previous studies have considered decorrelation as a geophysical phenomenon and integrated it with field surveys, for instance in mapping surface ruptures and estimating areas of major building damage caused by earthquakes (Simons et al., 2002; Fielding et al., 2005).

We also estimated surface displacement using InSAR, which can produce a surface displacement map over wide areas using phase information from paired satellite observations, without the need for ground-based observations. InSAR analysis has been successfully applied to understanding synoptic surface displacement caused by the 2011 earthquake (Kobayashi et al., 2011; Feng et al., 2012). These studies mainly focus on deformation over a few to several tens of kilometers. Here, the focus is on the detection of small-scale surface displacement produced by soil liquefaction, using InSAR analysis. 

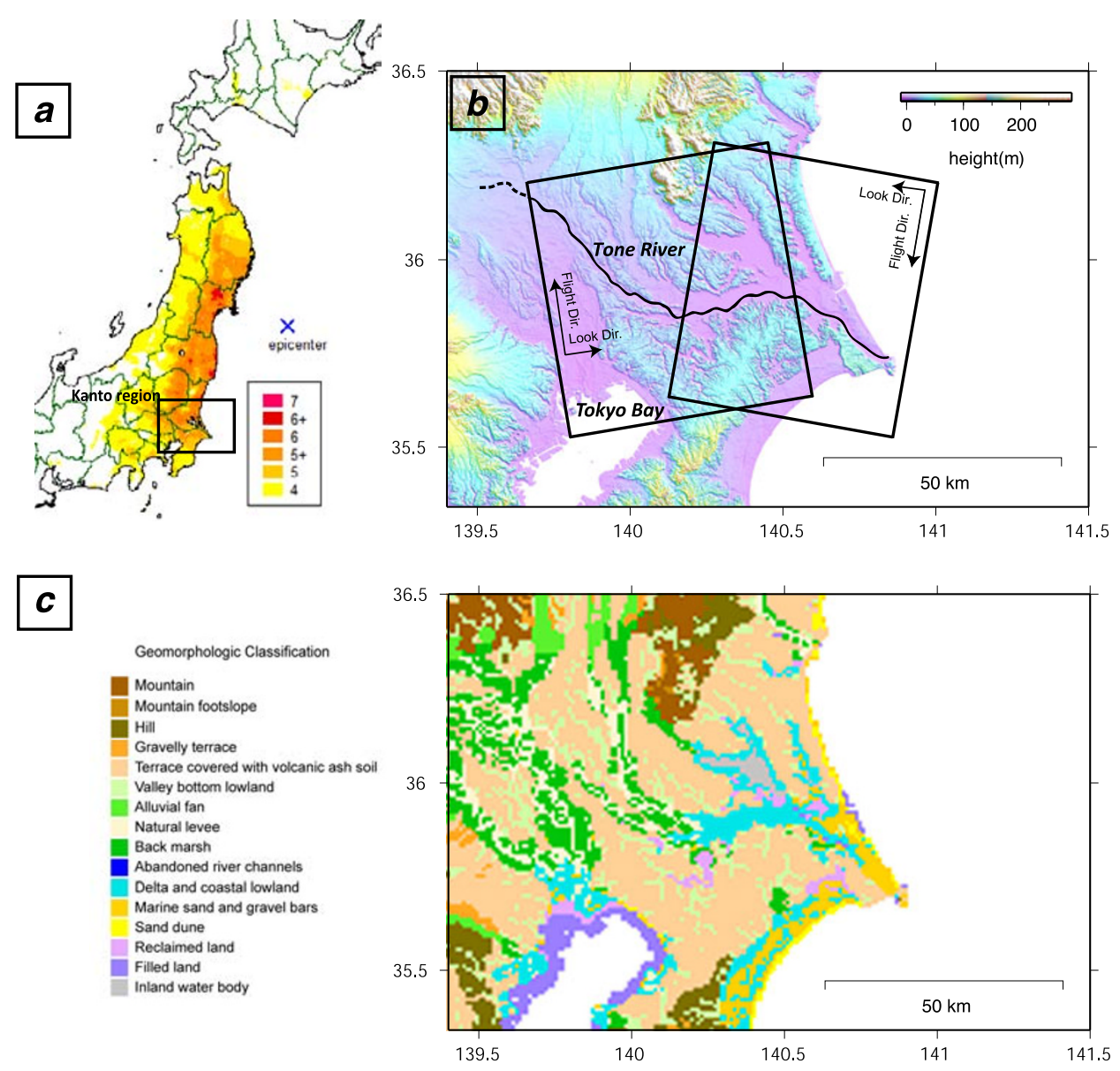

Fig. 1. (a) The study area (black rectangle) overlaid on the Japan Meteorological Agency seismic intensity of the 2011 earthquake (based on Hoshiba $e$ t al., 2011). (b) The topography of the Kanto region and the analyzed scene. Two rectangles located on the waterfront along Tokyo Bay and the Tone River covered by the SAR data, with ascending and descending orbits. Main branch of the Tone River is shown by a black line. (c) A geomorphologic classification map of the Kanto region with about a 1-km mesh (based on Wakamatsu et al., 2005).

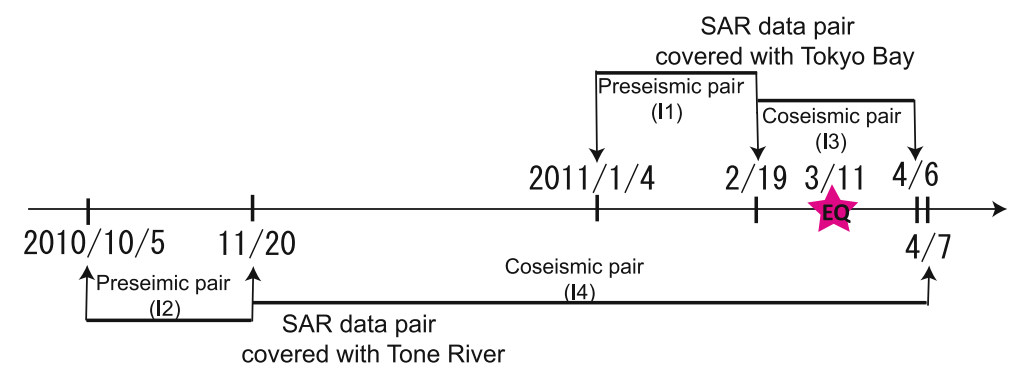

Fig. 2. Epochs of the SAR data used in this study. We used four SAR data pairs (I1-I4). Two pairs (preseismic pair I1; coseismic pair I3) include the waterfront along Tokyo Bay, and the other two pairs (preseismic pair I2; coseismic pair I4) include the waterfront along midstream and downstream of the Tone River. All data pairs (I1-I4) are used to estimate the change in the surface scattering property using the phase-corrected coherence, while two coseismic pairs (I3, I4) are used to estimate the local surface displacement associated with the soil liquefaction. A pink star represents the occurrence of the 2011 Tohoku earthquake.

\section{Method}

\subsection{Interferometric processing}

We obtained single-look complex (SLC) data after preprocessing SAR data from a Level-1.0 product. The SAR data were acquired by the Phased Array Type L-band SAR (PALSAR) instrument aboard the Japanese Advanced Land Observing Satellite (Table 1; Figs. 1(b) and 2). SLC data is composed of a regular grid with complex values $(C)$, which is decomposed into amplitude and phase information:

$$
C=A \exp (i \phi),
$$

where $A$ and $\phi$ represent amplitude and phase, respectively. Interferometric processing uses two SLC datasets, called master and slave. After repositioning and resampling each slave pixel to its corresponding master, we apply the following equation at each pixel:

$$
C_{1} C_{2}^{*}=A_{1} A_{2} \exp \left(i\left(\phi_{1}-\phi_{2}\right)\right),
$$

where $C_{1}$ and $C_{2}$ represent master and slave SLC data, and $*$ indicates complex conjugation. Equation (2) yields amplitude $\left(A_{1}, A_{2}\right)$ and phase $\left(\phi_{1}, \phi_{2}\right)$ information that 
Table 1. Interferogram and SAR data used. Master and slave indicate the period of SAR data for performing SAR interferometry. Displacement from master to slave is estimated via InSAR analysis. Off-nadir and Bperp show representative values of the off-nadir angle and perpendicular component of baseline, respectively.

\begin{tabular}{ccccccccc}
\hline Type & ID & Master & Slave & Cycles & Direction & Off-nadir & Bperp & Covered area \\
\hline Preseismic & I1 & 20110104 & 20110219 & 1 cycle & Ascending & $34.3^{\circ}$ & $709 \mathrm{~m}$ & Tokyo Bay \\
Preseismic & I2 & 20101005 & 20101120 & 1 cycle & Descending & $34.3^{\circ}$ & $593 \mathrm{~m}$ & Tone River \\
Coseismic & I3 & 20110219 & 20110406 & 1 cycle & Ascending & $34.3^{\circ}$ & $394 \mathrm{~m}$ & Tokyo Bay \\
Coseismic & I4 & 20101120 & 20110407 & 3 cycles & Descending & $34.3^{\circ}$ & $914 \mathrm{~m}$ & Tone River \\
\hline
\end{tabular}

describe the relationship between the SAR data in the pair.

We sought to detect the distribution of soil liquefaction using the change in coherence of amplitude $A$, and to estimate the ground displacement in the InSAR analysis using phase information $\phi$. Data processing was done using GAMMA software (Wegmüller and Werner, 1997). By averaging three pixels in the range direction, and five in the azimuthal direction, with multi-look processing, per-pixel resolution of the interferogram corresponds to a $14 \times 16-\mathrm{m}$ polygon in slant-range and azimuthal direction.

\subsection{Damage area detection using decorrelation}

The SAR system transmits microwaves and receives backscatter signal from illuminated surfaces. Generally, various scatterers (e.g., bare soil, buildings or crops) exist within each SAR data pixel. Reflective intensity (amplitude) and phase in a pixel is measured as the result of coherent summation of all returns from individual scatterers. When scatterers change between observations, the sum of scatter returns varies, which leads to decorrelation. To quantify decorrelation, we calculated the phase-corrected coherence $\gamma$ (Hagberg et al., 1995; Guarnieri and Prati, 1997):

$$
\gamma=\frac{\left|\sum_{n=1}^{N} C_{1}^{(n)} C_{2}^{*(n)} \exp \left(-i\left(\phi_{1}^{(n)}-\phi_{2}^{(n)}\right)\right)\right|}{\sqrt{\sum_{n=1}^{N}\left|C_{1}^{(n)}\right|^{2} \sum_{n=1}^{N}\left|C_{2}^{(n)}\right|^{2}}}
$$

where $C_{1}^{(n)}$ and $C_{2}^{(n)}$ indicate complex signals in each pixel and $\left(\phi_{1}^{(n)}-\phi_{2}^{(n)}\right)$ is the phase difference between master and slave. $N$ is the number of adjacent pixels used to compute coherence as a spatial average, usually called the window size. This size should be large enough to estimate coherence, because of computing the expectation as spatial averages over a number of pixels in an interferometric pair (Touzi et al., 1999; Zebker and Chen, 2005). However, spatial resolution decreases with window size. To estimate coherence, we chose $N=7 \times 7$, corresponding to an area roughly $98 \times 112 \mathrm{~m}$. This is almost the same as the number used by Fielding et al. (2005). Generally, coherence including both amplitude and phase is used in InSAR analysis. However, this coherence is influenced by phase variation not only from a change of noise in the signal, but also from systematic phase variation caused by topographic, atmospheric, or deformation gradients. To quantify and classify changes of surface properties attributable to liquefaction, we used phase-corrected coherence calculated based on amplitude. Amplitude in an SAR pixel represents backscatter intensity at a surface location. This intensity is affected by water content and surface roughness, given the same incidence angle, polarization, and radar wavelength. Hence, sand boils on the surface or buildings tilted by soil liquefaction alter amplitude relationships among adjacent pixels, generating decorrelation.

Although these surface changes are a source of decorrelation resulting from the earthquake, such decorrelation includes other effects that must be taken into account. Coherence comprises contributions from three effects (Zebker and Villasenor, 1992):

$$
\gamma=\gamma_{N} \gamma_{G} \gamma_{T}
$$

where $\gamma_{N}$ represents noise in the radar system and processing approach, $\gamma_{G}$ is geometric coherence proportional to the perpendicular component of the baseline, and $\gamma_{T}$ is the influence of temporal backscatter change, e.g. from surface cover change or vegetation.

Because soil liquefaction appears as a decrease in $\gamma_{T}$, we need to extract $\gamma_{T}$ from the total coherence. Therefore, we made a coherence difference map. This used the result of subtracting the coherence of preseismic data pairs (dataset I1 covers Tokyo Bay and I 2 covers Tone River; Table 1 and Fig. 2) from that of coseismic pairs, acquired before and after the earthquake (dataset I3 covers Tokyo Bay and I4 covers Tone River; Table 1 and Fig. 2):

$$
\gamma_{\text {diff }}=\gamma^{c}-\gamma^{p}=\gamma_{N} \gamma_{G}\left(\gamma_{T}^{c}-\gamma_{T}^{p}\right),
$$

where $\gamma^{c}$ and $\gamma^{p}$ are the coherence of the coseismic pair and preseismic pair, respectively.

This procedure is based on the assumption that components $\gamma_{N}$ and $\gamma_{G}$ are identical, or nearly identical, in both preseismic and coseismic pairs, for the following reasons. Since SAR data are obtained by the same sensor (PAL$\mathrm{SAR}), \gamma_{N}$ is identical. Supposing observation in a flat area and an uniform scatterers distribution within a pixel, $\gamma_{G}$ of the datasets used can be calculated from the value of perpendicular baselines and critical baseline of PALSAR (about $23000 \mathrm{~m}$ ). As a result, we obtained $\gamma_{G}$ values 0.97 for I1, 0.97 for I2, 0.98 for I3, and 0.96 for I4. These results show that the effect of $\gamma_{G}$ is almost identical.

Although soil liquefaction decreases $\gamma_{T}$, so does ordinal surface cover change and vegetation. To obtain a significant change of $\gamma_{T}$ caused only by the earthquake, we determined a coherence change threshold from the average and standard deviation of ordinal change in temporal coherence (Eq. (6)). This estimation was made using datasets acquired before the earthquake, between 2006 and 2011 (Table 2):

$$
\gamma_{\text {diff }}^{\text {thre }}=\gamma_{\text {diff }}^{\text {ave }}-k \gamma_{\text {diff }}^{\text {std }} \text {, }
$$

where $\gamma_{\text {diff }}^{\text {thre }}$ is the threshold of significant coherence difference caused by the earthquake at a pixel, and $\gamma_{\text {diff }}^{\text {ave }}$ and $\gamma_{\text {diff }}^{\text {std }}$ 
Table 2. SAR datasets used to estimate general trends of temporal coherence change before earthquake.

\begin{tabular}{cccrc}
\hline Master & Slave & Cycles & Bperp & Covered area \\
\hline 20060808 & 20060923 & 1 cycle & $576 \mathrm{~m}$ & Tokyo Bay \\
20071227 & 20080211 & 1 cycle & $945 \mathrm{~m}$ & Tokyo Bay \\
20080211 & 20080328 & 1 cycle & $96 \mathrm{~m}$ & Tokyo Bay \\
20081113 & 20081229 & 1 cycle & $22 \mathrm{~m}$ & Tokyo Bay \\
20081229 & 20090213 & 1 cycle & $740 \mathrm{~m}$ & Tokyo Bay \\
20100216 & 20100403 & 1 cycle & $144 \mathrm{~m}$ & Tokyo Bay \\
20110104 & 20110219 & 1 cycle & $709 \mathrm{~m}$ & Tokyo Bay \\
20080329 & 20080514 & 1 cycle & $146 \mathrm{~m}$ & Tone River \\
20080629 & 20080814 & 1 cycle & $1507 \mathrm{~m}$ & Tone River \\
20090817 & 20091002 & 1 cycle & $236 \mathrm{~m}$ & Tone River \\
20091002 & 20091117 & 1 cycle & $237 \mathrm{~m}$ & Tone River \\
20100520 & 20100705 & 1 cycle & $139 \mathrm{~m}$ & Tone River \\
20100705 & 20100820 & 1 cycle & $173 \mathrm{~m}$ & Tone River \\
20101005 & 20101120 & 1 cycle & $593 \mathrm{~m}$ & Tone River \\
20071228 & 20080514 & 3 cycles & $616 \mathrm{~m}$ & Tone River \\
20090517 & 20091002 & 3 cycles & $654 \mathrm{~m}$ & Tone River \\
20091002 & 20100102 & 3 cycles & $587 \mathrm{~m}$ & Tone River \\
\hline
\end{tabular}
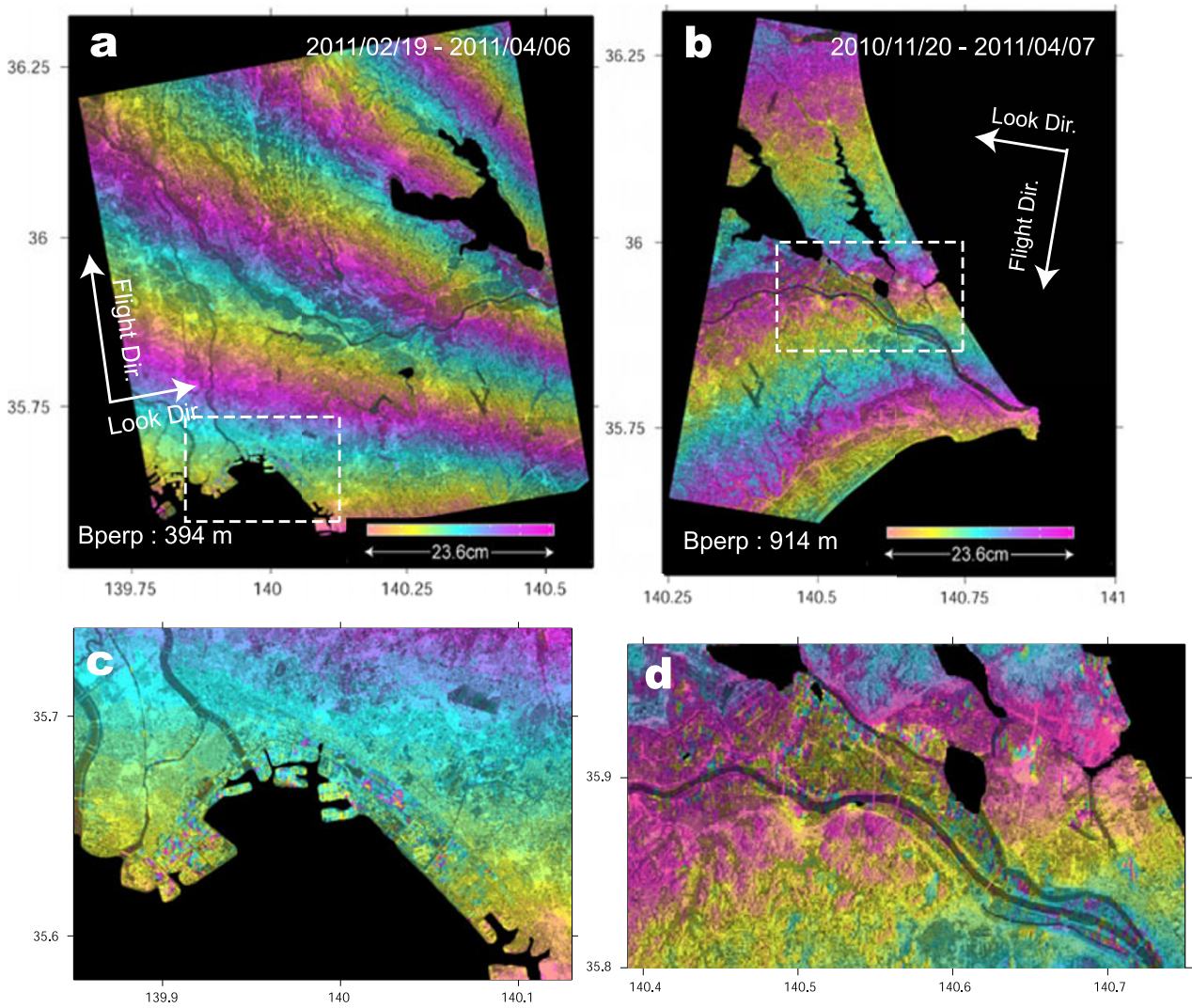

Fig. 3. Differential interferograms representing the regional trend of the surface displacement caused by the 2011 Tohoku earthquake, in each line of sight (LOS) direction. (a) A differential interferogram calculated from the coseismic pair I3 in Table 1 and Fig. 2 . (b) A differential interferogram calculated from the coseismic pair I4 in Table 1 and Fig. 2. (c) and (d) are the enlarged areas represented by the insets (dashed lines) in (a) and (b), in which the local phase disturbance is evident.

are the average and standard deviation of coherence difference calculated by preseismic datasets (Table 2). $k$ is a coefficient that represents a weighting factor for standard deviation. Suppose that the coherence difference at each pixel obeys a normal distribution (random surface cover change), we assumed that the coherence difference beyond 3-sigma $(k=3)$ represents significant surface change due to liquefaction.

\subsection{InSAR analysis}

From interferometric phase information, InSAR analysis enables us to obtain surface displacements. We used the coseismic pair datasets I3 (Tokyo Bay) and I4 (Tone River) in this analysis.

To remove the effect of the difference in satellite positions for the two acquisitions of a pair, we used 50-m mesh digital elevation models provided by the Geospatial 

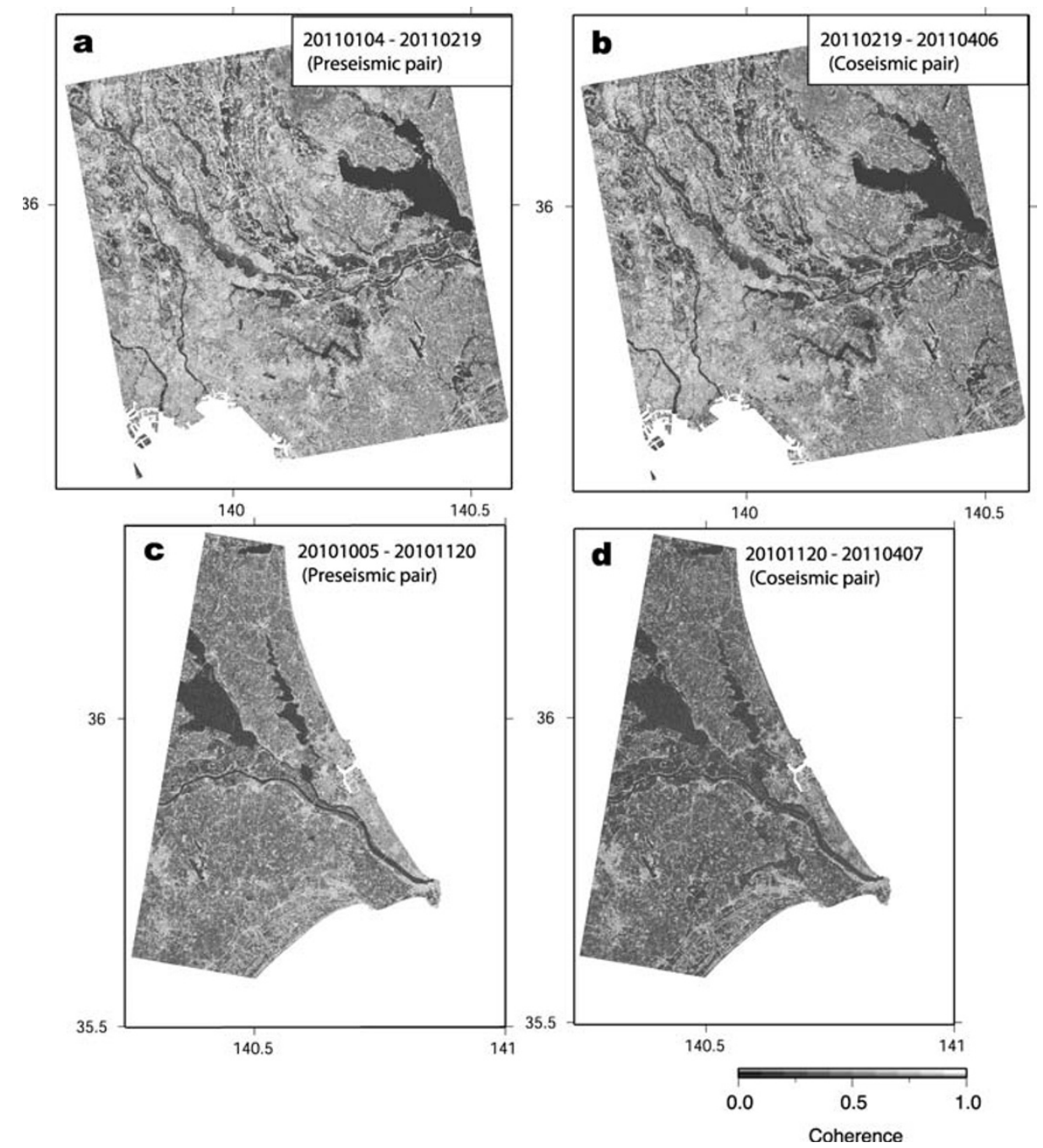

Fig. 4. (a) Coherence maps from the preseismic pair, covering Tokyo Bay (I1 in Table 1), (b) the coseismic pair, covering Tokyo Bay (I3 in Table 1), (c) the preseismic pair, covering the Tone River (I2 in Table 1), (d) the coseismic pair, covering the Tone River (I4 in Table 1).

Information Authority of Japan. To retain the high resolution of the data, we used an adaptive filter whose performance magnitude depends on coherence (Baran et al., 2003). Then, from phase information over the coherence threshold (0.30), we carefully conducted a phase unwrapping procedure using a branch-cut algorithm (Goldstein et al., 1988). A variety of displacement scales may be induced by soil liquefaction, and some spatially-small displacements may be filtered out or be eliminated by the coherence threshold. This filtering and unwrapping effect gives a spatially-continuous displacement, which is difficult to obtain from a field survey only. Finally, we obtained a differential interferogram that represents the total surface displacement caused by the 2011 earthquake along the line of sight (LOS) direction (Fig. 3). To display the local ground subsidence associated with soil liquefaction, we removed the long-wave ground displacement caused by the earthquake via the application of a best-fit quadratic function.

\section{Results and Discussion \\ 3.1 Temporal decorrelation}

Coherence maps (Fig. 4 and I1, I2, I3 and I4 in Fig. 6) show coherence reduction from three effects (Eq. (4)) between each pair. In the coherence difference maps of Fig. 5(a,b), zero values indicate areas where the coherence did not change between preseismic and coseismic pairs (that is, coherence in both interferograms was either high or low). Negative values indicate areas where coherence decreased in the coseismic pair (I3 or I4) relative to the preseismic pair (I1 or I2). Positive values indicate areas where coherence of the preseismic pair was lower than that of the coseismic pair. Negative values are distributed over the ground with artificial fill along Tokyo Bay (Fig. 5(a)), and near the banks of the Tone River (Fig. 5(b)). These observations suggest that physical properties of the ground surface, such as water content or surface roughness, were changed by the earthquake in these areas. Although negative values in the coherence difference map are possibly associated with the earthquake, we must discriminate scatter change caused by the 2011 earthquake from other temporal effects (e.g., ordinal change in surface cover or decorrelation from vegetation). Therefore, we estimated the coherence change threshold at 

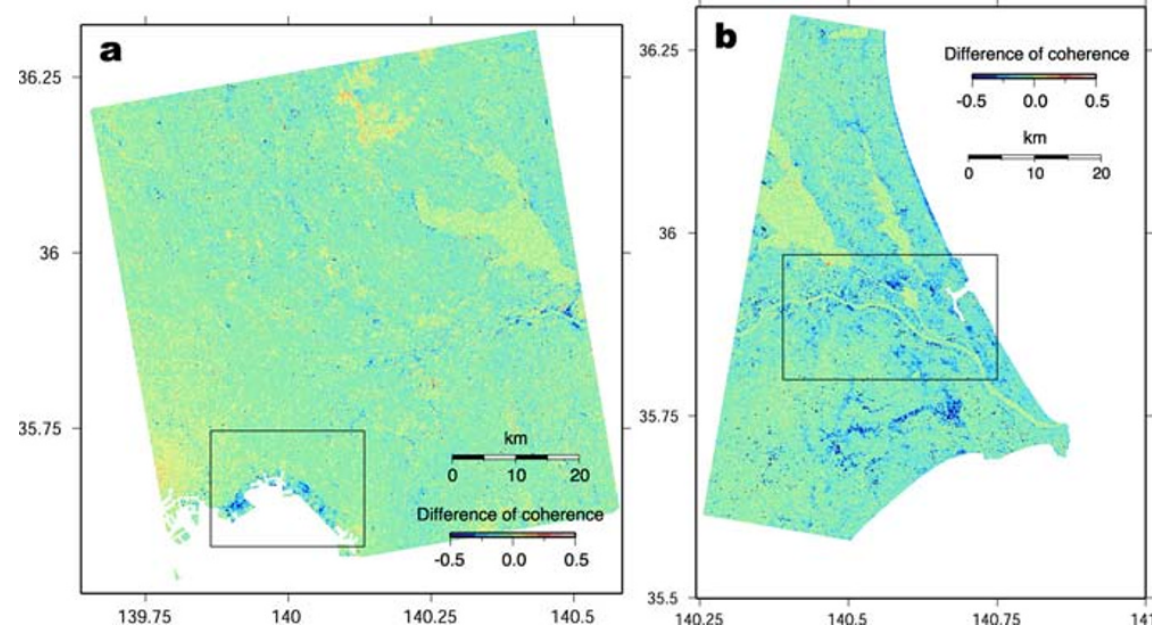

Fig. 5. Difference maps of the phase-corrected coherence (i.e., the coherence difference maps) between (a) I1 and I3 (covering the waterfront along Tokyo Bay), and (b) I2 and I4 (covering the waterfront along the midstream and downstream of the Tone River).
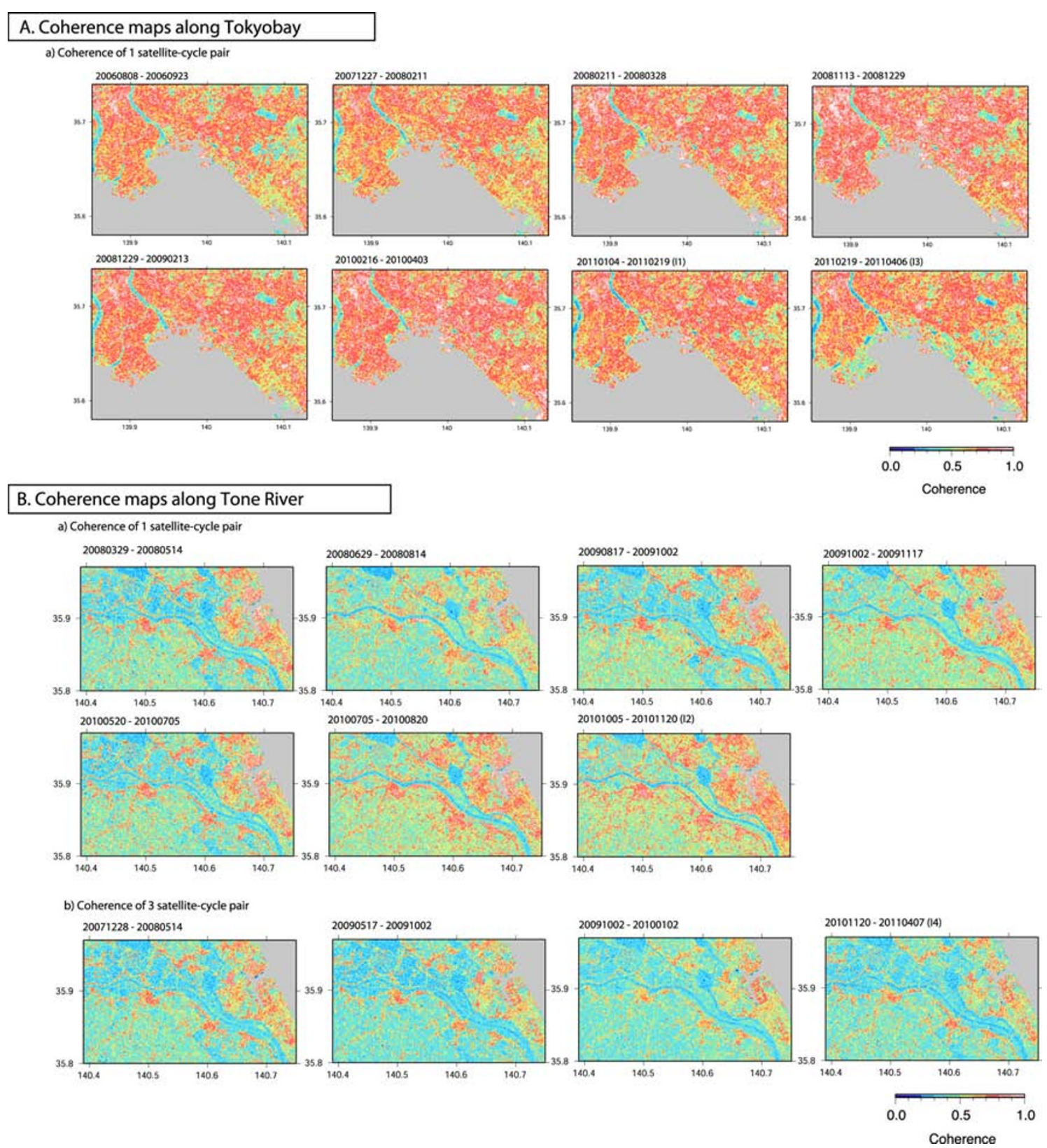

Fig. 6. Coherence maps of each dataset used for areas along (A) Tokyo Bay, and (B) the Tone River. 


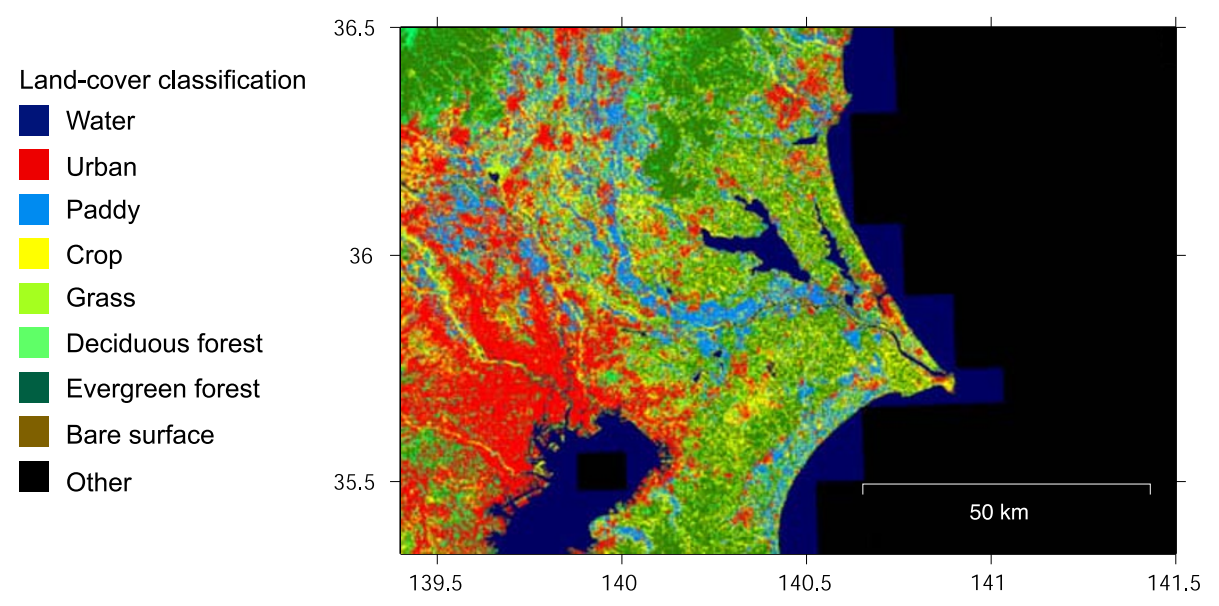

Fig. 7. Land-cover classification derived from satellite optical data (based on Takahashi et al., 2010, 2012).
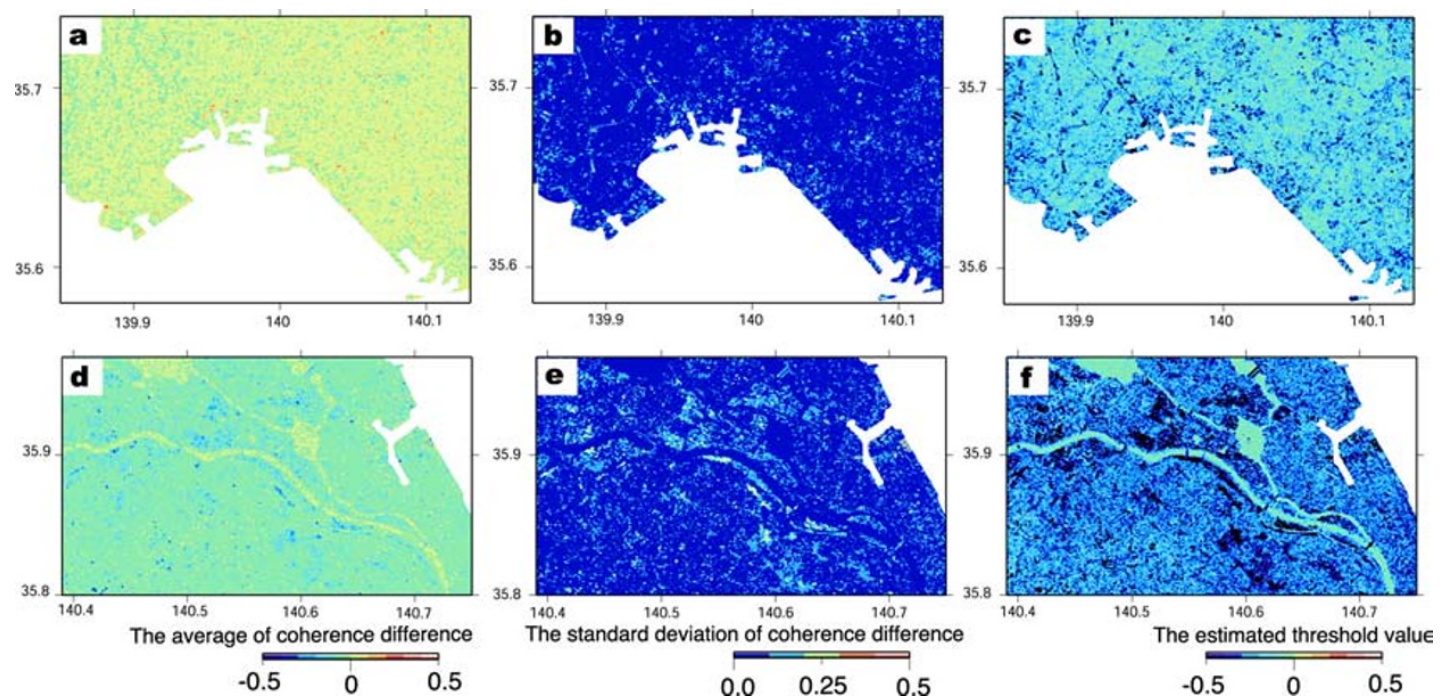

Fig. 8. (a) Averages and (b) standard deviations of coherence values, and (c) estimated threshold values of the coherence difference calculated from the preseismic pair in the areas along Tokyo Bay. (d) Averages and (e) standard deviations of coherence values, and (f) estimated threshold values of the coherence difference calculated from the preseismic pair in the areas along the Tone River.

each pixel using the average and standard deviation of the coherence change caused by other temporal effects, which appears in the coherence difference maps.

To reveal the effects of the temporal and spatial baseline conditions of our analyzed area, we studied datasets before the earthquake within a perpendicular baseline of $2000 \mathrm{~m}$ (Table 2): one satellite-cycle between 2006 and 2011 covering waterfront areas along Tokyo Bay (seven datasets), and one cycle (seven datasets) and three cycles (three datasets) for the same period along the Tone River. Coherence maps of each preseismic pair showed that most of the coherence in the areas along Tokyo Bay was stable with time, while the coherence along the Tone River was low and variable (Fig. 6). This can be interpreted as a result of surface cover in each area. Land-cover classification derived from optical satellite data (Takahashi et al., 2010, 2012) show areas along the bay to be urban, mainly covered by buildings and asphalt roads. In contrast, areas along the Tone River are classified as paddy, crops and other vegetation, where backscatter signals tend to be unstable (Fig. 7). Ordinal coherence change in paddy and crop areas along the river were discovered in the analysis (Fig. 6(B)). Along the river, three-cycle pair coherences are lower than those of onecycle pairs, because surface cover likely changes with the duration of observation (Fig. 6(B)). Considering the physical reason for decorrelation in vegetated and agriculture areas (i.e., surface cover change), it may be inferred that there are annual trends of coherence reduction at each pixel. However, since the observation data are limited, we used all one-cycle and three-cycle pairs from areas along the Tone River for further analysis.

Next, average and standard deviation of coherence difference values were calculated using all dataset combinations for the Tokyo Bay area (21 total combinations), and onecycle pair and three-cycle pair combinations for the Tone River area (21 total combinations) (Fig. 8(a, b, d, e)). Then, according to Eq. (6), we calculated the coherence change threshold at each pixel. We considered a coherence difference below the threshold as a significant decorrelation caused by surface changes of the 2011 earthquake (Fig. 8(c, f)). The processing indicated that the threshold in areas along the bay is nearly zero, because the coherence is stable 

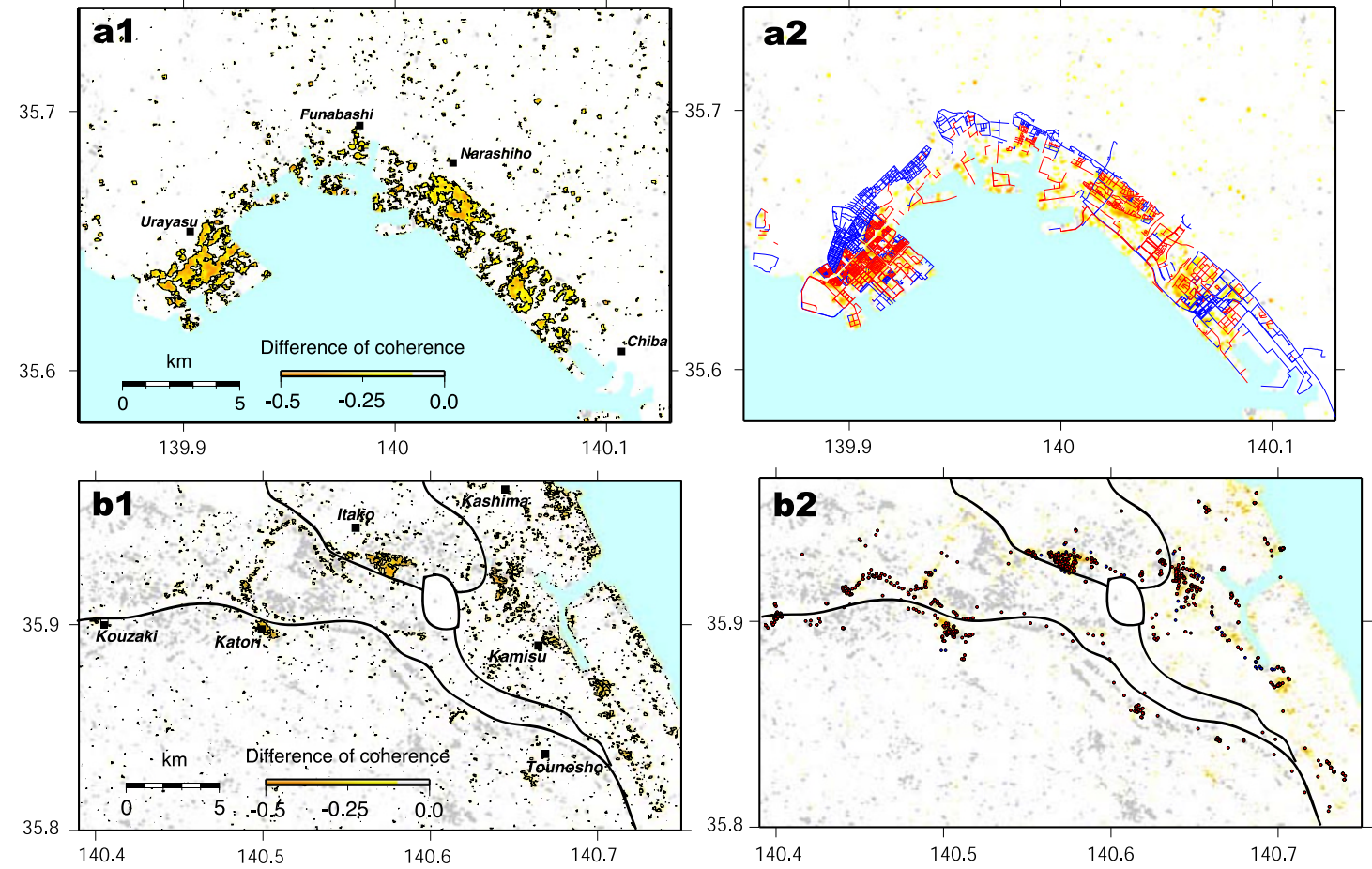

Fig. 9. Estimated liquefaction areas along (a1) Tokyo Bay and (b1) the Tone River. Observed liquefaction through field surveys conducted by KRDB and JGS (2011) overlaid on the estimated liquefaction for the waterfronts along (a2) Tokyo Bay and (b2) the Tone River. Red and blue indicate liquefaction and non-liquefaction areas, respectively, detected by field survey. Yellow and orange areas indicate the negative coherence areas below the coherence reduction threshold value. Significant coherence reduction cannot be estimated in gray areas where coherence in preseismic pair is less than the threshold value. Bold black lines indicate the Tone River.
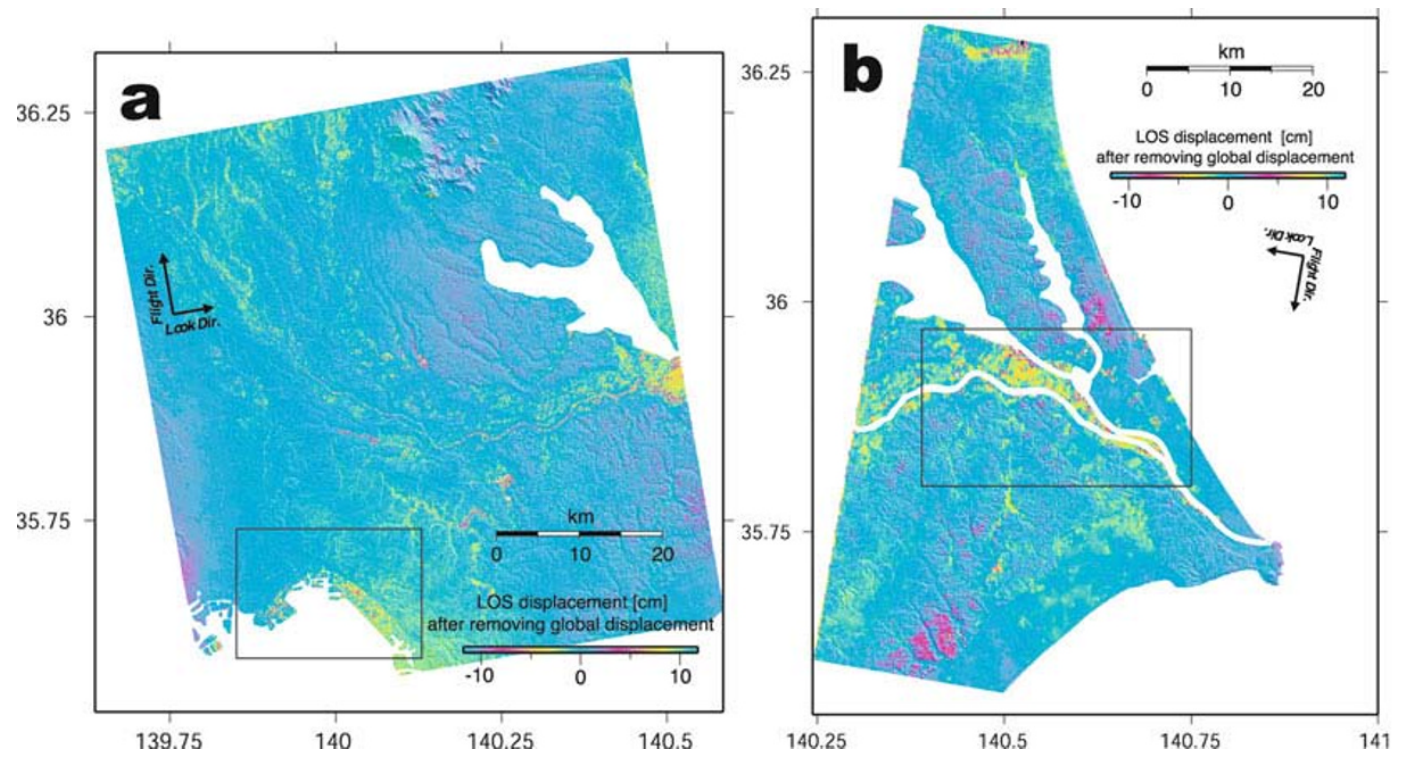

Fig. 10. Local surface displacements after the removal of the regional displacement trend obtained from (a) I3 (covering the waterfront along Tokyo Bay), and (b) I4 (covering the waterfront along the midstream and downstream of the Tone River).

over time, as described previously (Fig. 6(A)). On the other hand, the threshold in agricultural areas along the river is lower than in the urban area (Fig. 8(f)). This means that it is difficult to detect small surface changes caused by the earthquake in these areas. However, in the urban area along the Tone River, the coherence remains stable enough to detect surface changes (Fig. 8(d, e, f)).

To confirm this association of surface scatter changes with soil liquefaction, we compared our result with surveys conducted on foot, or based on aerial photography, soon after the earthquake (KRDB and JGS, 2011). The survey clarified soil liquefaction areas in the Kanto region, checking the existence of sand boils or water spouts associated with the liquefaction. The walking survey was mainly performed along roads, and inaccessible areas such as industrial plants were covered by aerial photography. These are plotted in Fig. 9(a2, b2), using red and blue to indicate liquefaction and non-liquefaction locations, respectively. Yel- 

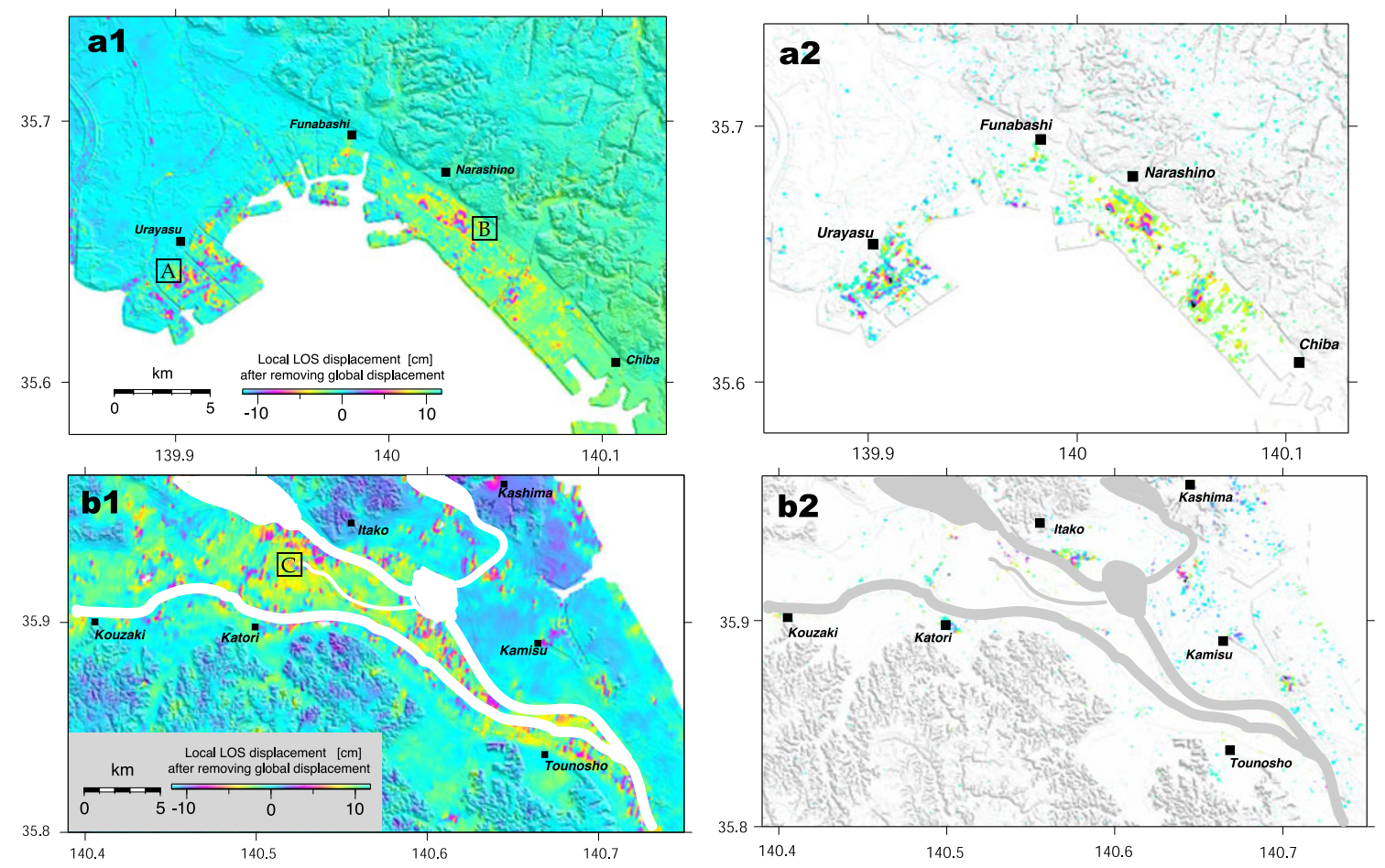

Fig. 11. Local surface displacements near the waterfront along (a1) Tokyo Bay and (b1) the Tone River. Local surface displacements in the liquefaction areas along (a2) Tokyo Bay and (b2) the Tone River.

low and orange areas in Fig. 9(a1, a2, b1, b2) indicate negative values of phase-corrected coherence, under the estimated threshold. Significant decorrelation due to disaster in areas where the coherence of the preseismic pair is lower than the threshold value cannot be detected in principle. These areas are plotted in gray in Fig. 9(a1, a2, b1, b2). Comparison shows that $85 \%$ and $61 \%$ of liquefaction locations detected by the field survey of KRDB and JGS (2011) in areas along Tokyo Bay and the Tone River, respectively, are consistent with the present results. Because of this consistency, negative coherence corresponds to surface changes from soil liquefaction caused by the 2011 earthquake. Agreement in areas along the bay is slightly greater than along the river, probably because coherence was temporally stable in those areas (Fig. 6), resulting in easy detection of coherence change.

\subsection{InSAR-derived surface displacement}

Figures 10 and 11 show local surface displacement inferred by InSAR, after the removal of global surface displacement. Magnitudes between about -30 to $3 \mathrm{~cm}$ were detected in the soil liquefaction areas along Tokyo Bay and the Tone River. Although local changes in the positive range are also found outside the soil liquefaction areas, we cannot completely determine whether these changes reflect surface displacement or other effects (e.g., quadratic fitting error or atmospheric artifacts) (Fig. 10). Nevertheless, the magnitude of these changes (less than $3 \mathrm{~cm}$ ) is much smaller than that of local surface displacement in soil liquefaction areas. Previous studies have shown that decorrelation leads to an increase of phase variance (Rodriguez and Martin, 1992; Just and Bamler, 1994). In the soil liquefaction areas, there are certainly decorrelation-induced phase discontinuities (e.g., A in Fig. 11(a1), C in Fig. 11(b1)); however, we partly obtained a spatially-continuous displacement (e.g., B in Fig. 11(a1)). Since most of the SAR data in liquefaction areas were collected in only one direction (not both ascending and descending orbits), we cannot explicitly determine whether these local surface displacements were subsidence. We inferred that local displacement may be ground subsidence, because soil liquefaction can cause soil grains to rearrange into a more dense packing due to the phenomena that a mass of soil loses its shear resistance and pore water pressures increase, and such displacement is at liquefaction locations where the ground surface is flat.

Estimated local ground displacements demonstrate characteristics of local displacement in each area. Along Tokyo Bay, this displacement was particularly widespread and continuous in the Narashino area (B in Fig. 11(a1)). A continuous fringe pattern predominates in the Narashino (B in Fig. 11(a1)) and Chiba areas, whereas a discontinuous fringe pattern dominates in the Urayasu area (A in Fig. 11(a1)). These observations suggest that continuous fringe pattern areas are uniformly deformed over a relatively wide area, but this is not true of discontinuous areas.

\section{Conclusions}

We detected changes of surface scattering properties caused by the 2011 Tohoku earthquake, using the difference of phase-corrected coherence between preseismic and coseismic SAR data pairs. To retrieve the significant decorrelation signal attributable to the earthquake, we proposed the use of a coherence change threshold estimated from preseismic datasets. We also revealed local surface displacements using InSAR analysis. The results are consistent with field surveys. Therefore, these surface changes are associated with soil liquefaction. The areal extent of soil lique- 
faction inferred from our analysis is difficult to obtain from ground-based measurements. This study shows that SAR data is effective for investigating the extent and detail of soil liquefaction.

In selecting the SAR data, Massonnet and Feigl (1998) reported that the maximum detectable deformation gradient is one fringe per pixel. Thus, long-wavelength, highresolution SAR data are better for detecting high deformation gradients associated with soil liquefaction. This means that L-band SAR data are superior to C-band and X-band data, given the same spatial resolution. We performed SAR interferometry analysis and then compared the result with field survey results. However, InSAR analysis carried out immediately after an earthquake shows promise in effectively guiding subsequent field surveys.

Acknowledgments. The PALSAR data used in this study belong to the Ministry of Economy, Trade and Industry (METI) and the Japan Aerospace Exploration Agency (JAXA). Level1.0 data were processed and provided by the Earth Remote Sensing Data Analysis Center (ERSDAC). We also acknowledge the Kanto Regional Development Bureau of the Ministry of Land, Infrastructure, Transport and Tourism (KRDB) and the Japanese Geotechnical Society (JGS) for providing soil liquefaction data. The land cover map was produced and furnished by the Earth Observation Research Center, Japan Aerospace Exploration Agency (JAXA/EORC). We also express our appreciation to two reviewers (Dr. Taku Ozawa and anonymous) for their insightful comments, which helped us improve this paper. The authors acknowledge support for this study from ERSDAC and the Global Center for Education and Research on Human Security Engineering for Asian Megacities (GCOE). T. Tsuji gratefully acknowledges the support of the International Institute for Carbon Neutral Energy Research (I2CNER), sponsored by the World Premier International Research Center Initiative (WPI), MEXT, Japan.

\section{References}

Atzori, S., C. Tolomei, A. Antonioli, J. P. M. Boncori, S. Bannister, E. Trasatti, P. Pasquali, and S. Salvi, The 2010-2011 Canterbury, New Zealand, seismic sequence: Multiple source analysis from InSAR data and modeling, J. Geophys. Res., 117, B08305, 2012.

Baran, I., M. P. Stewart, B. M. Kampes, Z. Perski, and P. Lilly, A modification to the Goldstein radar interferogram filter, IEEE Trans. Geosci. Remote Sens., 41, 2114-2118, 2003.

Bhattacharya, S., M. Hyodo, K. Goda, T. Tazoh, and C. A. Taylor, Liquefaction of soil in the Tokyo Bay area from the 2011 Tohoku (Japan) earthquake, Soil Dyn. Earthq. Eng., 31, 1618-1628, 2011.

Bürgmann, R., P. A. Rosen, and E. J. Fielding, Synthetic aperture radar interferometry to measure Earth's surface topography and its deformation, Ann. Rev. Earth Planet. Sci., 28, 169-209, 2000.

Feng, G., X. Ding, Z. Li., J. Mi, L. Zhang, and M. Omura, Calibration of an InSAR-derived coseismic deformation map associated with the 2011 Mw-9.0 Tohoku-oki Earthquake, IEEE Trans. Geosci. Remote Sens. Lett., 9, 302-306, 2012.

Fielding, E. J., M. Talebian, P. A. Rosen, H. Nazari, J. A. Jackson, M. Ghorashi, and R. Walker, Surface ruptures and building damage of the 2003 Bam, Iran, earthquake mapped by satellite synthetic aperture radar interferometric correlation, J. Geophys. Res., 110, B03302, 2005.

Goldstein, R. M. and C. L. Werner, Radar Interferogram Filtering for Geophysical Applications, Geophys. Res. Lett., 25, 4035-4038, 1998.

Goldstein, R. M., H. A. Zebker, and C. L. Werner, Satellite radar interferometry: Two-dimensional phase unwrapping, Radio Sci., 23, 713-720, 1988.
Guarnieri, A. M. and C. Prati, SAR interferometry: A "Quick and Dirty" coherence estimator for data browsing, IEEE Trans. Geosci. Remote Sens., 35, 660-669, 1997.

Hagberg, J. O., L. M. H. Ulander, and J. Askne, Repeat-pass SAR interferometry over forested terrain, IEEE Trans. Geosci. Remote Sens., 33, 331-340, 1995.

Hoshiba, M., K. Iwakiri, N. Hayashimoto, T. Shimoyama, K. Hirano, Y Yamada, Y. Ishigaki, and H. Kikuta, Outline of the 2011 off the Pacific coast of Tohoku Earthquake $\left(M_{\mathrm{w}} 9.0\right)$-Earthquake Early Warning and observed seismic intensity-, Earth Planets Space, 63, 547-551, 2011.

Just, D., and R. Bamler, Phase statistics of interferograms with applications to synthetic aperture radar, Appl. Opt., 33, 4361-4368, 1994.

Kanto Regional Development Bureau of Ministry of Land, Infrastructure, Transport and Tourism (KRDB) and Japanese Geotechnical Society (JGS), Investigation of soil liquefaction in Kanto region associated with the 2011 Tohoku earthquake (http://www.ktr.mlit.go.jp/ or http://www.jiban.or.jp/), 2011 (in Japanese).

Kobayashi, T., M. Tobita, T. Nishimura, A. Suzuki, Y. Noguchi, and M. Yamanaka, Crustal deformation map for the 2011 off the Pacific coast of Tohoku Earthquake, detected by InSAR analysis combined with GEONET data, Earth Planets Space, 63, 621-625, 2011.

Massonnet, D. and K. L. Feigl, Radar interferometry and its application to changes in the Earth's surface, Rev. Geophys. 36, 441-500, 1998.

Ozawa, S., T. Nishimura, H. Suito, T. Kobayashi, M. Tobita, and T. Imakiire, Coseismic and postseismic slip of the 2011 magnitude-9 TohokuOki earthquake, Nature, 475, 373-376, 2011.

Rodriguez, E. and J. M. Martin, Theory and design of interferometric synthetic aperture radars, IEE Proc. F, 139, 147-159, 1992.

Senna, S., N. Hasegawa, T. Maeda, and H. Fujiwara, Liquefaction damage of the Tonegawa basin caused by the 2011 off the pacific coast of Tohoku earthquake, Proceedings of the International Symposium on Engneering Lessons Learned from the 2011 Great East Japan Earthquake, 719-730, 2012.

Simons, M. and M. Rosen, Interferometric Synthetic Aperture Rader Geodesy, in Treatise on Geophysics, edited by G. Schubert, 391-446, Elsevier B.V, Netherlands, 2007.

Simons, M., Y. Fialko, and L. Rivera, Coseismic deformation from the 1999 Mw 7.1 Hector mine, California, Bull. Seismol. Soc. Am., 92, 1390-1402, 2002.

Takahashi, M., K. Nasahara, T. Tadono, H. Murakami, and G. Kadosaki, High accuracy land cover map by "Daichi" ALOS satellite, Proceedings of the 49th Annual Meeting of Remote Sensing Society of Japan, 41-42, 2010 (in Japanese with English abstract).

Takahashi, M., K. Nasahara, T. Tadono, T. Watanabe, M. Doutsu, T. Sugimura, and N. Tomiyama, Update of JAXA high resolution land-use and land-cover map and maintenance of ground validation data, Proceedings of the 52th Annual Meeting of Remote Sensing Society of Japan, 81-82, 2012 (in Japanese with English abstract).

Touzi, R., A. Lopes, J. Bruniquel, and P. W. Vachon, Coherence estimation for SAR imagery, IEEE Trans. Geosci. Remote Sens., 37, 135-149, 1999

Wakamatsu, K., S. Kubo, M. Matsuoka, K. Hasegawa, and M. Sugiura, Japan Engineering Geomorphologic Classification Map, 96 pp, University of Tokyo Press, Japan, 2005 (product serial number: JEGM0001).

Wegmüller, U. and C. L. Werner, Gamma SAR processor and interferometry software, Proceedings of the 3rd ERS Symposium, Eur. Space Agency Spec. Publ., ESA SP-414, 1686-1692, 1997.

Yasuda, S. and K. Harada, Liquefaction-induced Damage in the Reclaimed Lands along Tokyo Bay, J. Jpn. Geotech. Soc., 59, 38-41, 2011 (in Japanese with English abstract).

Zebker, H. A. and K. Chen, Accurate estimation of correlation in InSAR observations, IEEE Trans. Geosci. Remote Sens. Lett., 2, 124-127, 2005

Zebker, H. A. and J. Villasenor, Decorrelation in interferometric radar echoes, IEEE Trans. Geosci. Remote Sens., 30, 950-959, 1992.

K. Ishitsuka (e-mail: k_ishitsuka@earth.kumst.kyoto-u.ac.jp), T. Tsuji, and T. Matsuoka 